\title{
SÍNTESE E CARACTERIZAÇÃO ESTRUTURAL DO LIGANTE ISATINA-3-(N-BENZILTIOSSEMICARBAZONA) E DO SEU COMPLEXO DE MERCÚRIO(II)
}

\author{
Alexandra de Souza Fonseca, Gisele Louro Peres, Tomás Garcia Storino, Leandro Bresolin e Vanessa Santana Carratu* \\ Escola de Química e Alimentos, Universidade Federal de Rio Grande, Av. Itália, km 08, 96201-900 Rio Grande - RS, Brasil \\ Vinícius F. Giglio, Estela dos Reis Crespan e Manfredo Hörner \\ Departamento de Química, Centro de Ciências Naturais e Exatas, Universidade Federal de Santa Maria, Av. Roraima, s/n, Campus, \\ 97105-900 Santa Maria - RS, Brasil
}

Recebido em 21/8/09; aceito em 18/3/10; publicado na web em 29/6/10

\begin{abstract}
SYNTHESIS AND STRUCTURAL CHARACTERIZATION OF THE LIGAND ISATIN-3-(N4-BENZYLTHIOSEMICARBAZONE) AND ITS MERCURY(II) COMPLEX. The reaction of 4-(phenyl)thiosemicarbazide with isatin yielded a new ligand, isatin-3-(N4benzylthiosemicarbazone). Isatin-3-(N ${ }^{4}$-benzylthiosemicarbazone) deprotonated in ethanol/KOH reacts with an ethanolic solution of $\mathrm{Hg}\left(\mathrm{NO}_{3}\right)_{2}$ to give a mercury complex. The compounds were characterized by IR and X-ray single crystal structure determination. The X-ray studies revealed that the complex possesses a tetrahedral geometry with two deprotonated thiosemicarbazone ligands coordenated. The ligand and its mercury complex crystallize in the monoclinic $\left(P 2_{1} / c\right)$ and triclinic $(P-1)$ crystal system, respectively.
\end{abstract}

Keywords: mercury complex; thiosemicarbazone; hydrogen bonds.

\section{INTRODUÇÃO}

Compostos derivados de tiossemicarbazonas têm despertado especial interesse na investigação científica devido a suas propriedades químicas, biológicas e farmacológica. ${ }^{1-3}$ Estudos demonstram que tiossemicarbazonas derivadas de isatina possuem aplicações no tratamento contra varíola ${ }^{4}$ e derivados da isatina- $\beta$-tiossemicarbazona apresentam atividades anti-HIV e inibidora do vírus Encephalitis Japonese entre outros. ${ }^{5,6}$ Tiossemicarbazonas provenientes de vários aldeídos e cetonas ocupam lugar especial entre os ligantes orgânicos, uma vez que apresentam vários átomos doadores e diferentes modos de coordenação, dependendo dos reagentes de partida e das condições de reação para sua obtenção. ${ }^{7}$ Os derivados de tiossemicarbazonas são ótimos ligantes frente a metais de transição devido ao comportamento ácido do grupo NH imínico, que uma vez desprotonado provoca a deslocalização de carga ao longo da cadeia originando um ligante aniônico. ${ }^{8}$ Do ponto de vista puramente químico, estes ligantes se apresentam versáteis com ampla capacidade quelante, além da possibilidade de ocorrência de ligações de hidrogênio formando estruturas poliméricas. $\mathrm{O}$ estudo de cadeias supramoleculares é importante no contexto de suas aplicações como carreadores no transporte de drogas em sistemas biológicos, bem como na detecção de traços de metais. ${ }^{9,10}$

São descritas na literatura ${ }^{7}$ a preparação, caracterização espectroscópica e termogravimétrica de complexos do ligante isatina- $\beta$ tiossemicarbazona com vários metais, entre eles o mercúrio (II). Desta forma, a elucidação estrutural das moléculas aqui apresentadas é uma contribuição relevante ao estudo da química das tiossemicarbazonas.

\section{PARTE EXPERIMENTAL}

\section{Materiais e equipamentos}

Todos os reagentes utilizados são disponíveis comercialmente e foram usados sem tratamentos prévios (Sigma Aldrich, Vetec). O aparelho de ponto de fusão utilizado foi o modelo 430D Fisatom. Os espectros de absorção na região do infravermelho foram obtidos

*e-mail: vanessa_carratu@yahoo.com.br por refletância difusa no espectrofotômetro Shimadzu - IR Prestige-21. Todos os dados de difração de raios-x foram coletados no difratômetro Bruker CCD X8 Kappa Apex II com detector de área, sob temperatura ambiente. Os refinamentos das estruturas foram realizados com o programa SHELXL97 ${ }^{11}$ e a redução dos dados e correção de absorção envolveram os programas SAINT ${ }^{12}$ e SADA$\mathrm{BS},{ }^{12}$ respectivamente.

\section{Síntese do ligante isatina-3-(N+benziltiossemicarbazona)}

O ligante foi preparado mediante reação entre quantidades equimolares de isatina (1 mmol) e 4-feniltiossemicarbazida (1 mmol) dissolvidas em metanol e mantidas sob refluxo, em meio ácido, por 6 h. Após resfriamento, um produto sólido amarelo foi isolado mediante filtração simples, lavado com água destilada e seco. Os cristais foram isolados após recristalização e evaporação lenta do solvente (metanol). Rendimento $80 \%$ (238 mg) ponto de fusão $237^{\circ} \mathrm{C}$.

Síntese do complexo bis[isatina-3-(N4-

benziltiossemicarbazonato)] mercúrio (II)

O complexo foi preparado através da reação entre 2 mmols do ligante previamente desprotonado com hidróxido de potássio e 1 mmol de nitrato de mercúrio dissolvidos em etanol e mantidos sob agitação em temperatura ambiente por $3 \mathrm{~h}$. Os cristais laranja aptos à difração de raios-x foram isolados após lenta evaporação dos solventes em um ensaio de cristalização com a mistura 2:1 de tolueno/acetona. Ponto de fusão dos cristais $205^{\circ} \mathrm{C}$. O rendimento do complexo não foi estimado, por serem obtidos poucos cristais utilizados para as análises aqui apresentadas.

\section{RESULTADOS E DISCUSSÃO}

O composto isatina-3-( $\mathrm{N}^{4}$-benziltiossemicarbazona) (Figura 1) poderia atuar como ligante monodentado coordenando-se através do nitrogênio aminíco, como observado para um ligante semelhante na literatura, ${ }^{13}$ ou ainda como quelante tridentado coordenando-se através do nitrogênio iminíco, oxigênio carbonílico e enxofre do 
grupo tiolato. ${ }^{7}$ Porém, a estrutura do complexo de mercúrio (Figura 2) apresentada neste trabalho traz o ligante isatina-3- $\left(\mathrm{N}^{4}\right.$-benziltiossemicarbazona) atuando como quelante bidentado, ou seja, a ocorrência da coordenação através do átomo de oxigênio doador é dependente da natureza do metal ${ }^{4}$ e neste caso não é observada.

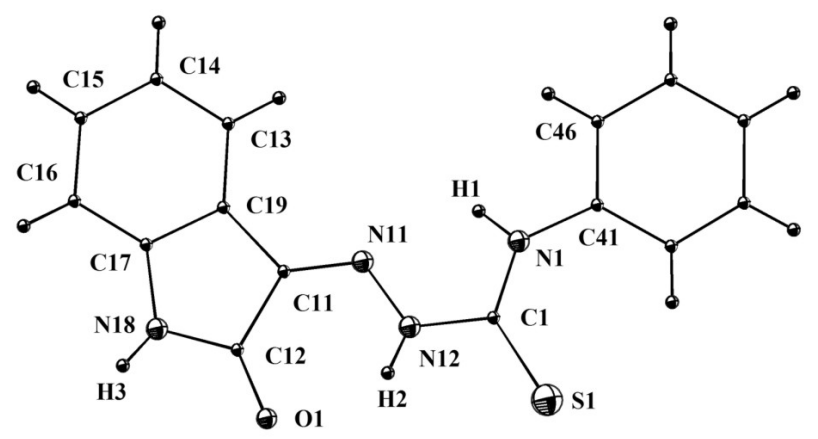

Figura 1. Estrutura de isatina-3-( $N^{4}$-benziltiossemicarbazona)

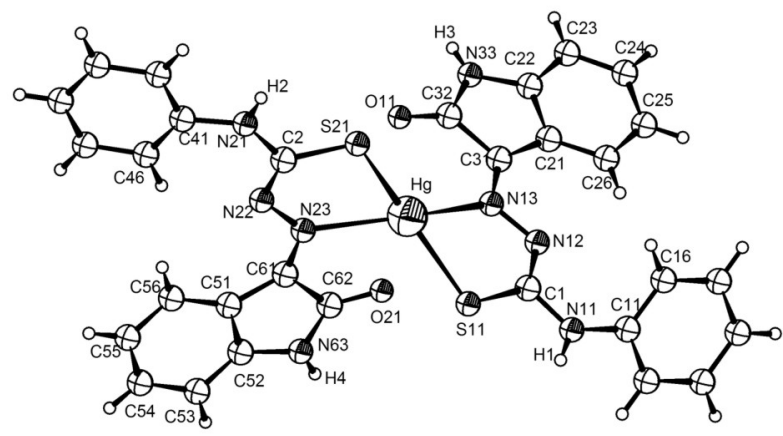

Figura 2. Estrutura do complexo bis[isatina-3-( $N^{4}$-benziltiossemicarbazonato)] mercúrio (II)

\section{Espectroscopia de infravermelho}

O espectro na região do infravermelho para o ligante isatina-3-( $\mathrm{N}^{4}-$ benziltiossemicarbazona) apresenta bandas intensas na região 3298-3178 $\mathrm{cm}^{-1}$ atribuídas às vibrações de estiramento $\mathrm{v}(\mathrm{N}-\mathrm{H})$ do composto neutro. Bandas em 3364-3181 $\mathrm{cm}^{-1}$ foram atribuídas às vibrações de estiramento $v(\mathrm{~N}-\mathrm{H})$ para 5 -fluor-isatina-3-(N-benziltiossemicarbazona). ${ }^{4}$ As bandas referentes a $v(\mathrm{C}=\mathrm{O})$ de cetonas aromáticas ocorrem na região entre $1700-1640 \mathrm{~cm}^{-1} \cdot{ }^{14-16}$ No espectro do ligante, a forte absorção em 1693 $\mathrm{cm}^{-1}$ é atribuída a $v(\mathrm{C}=\mathrm{O})$ e para a $v(\mathrm{C}=\mathrm{N}) 1593 \mathrm{~cm}^{-1}$. A identificação da $v(\mathrm{C}=\mathrm{S})$ é bastante difícil, pois o espectro no infravermelho apresenta várias bandas na região entre $1420-700 \mathrm{~cm}^{-1}{ }^{17}$ no presente trabalho as bandas em 1147 e $744 \mathrm{~cm}^{-1}$ são atribuídas as $v(\mathrm{C}=\mathrm{S})$ sendo que apenas a última é afetada pela coordenação ao centro metálico.

No complexo bis[isatina-3-( $\mathrm{N}^{4}$-benziltiossemicarbazonato)] mercúrio (II), onde a $v(\mathrm{O}-\mathrm{H})$ é proveniente das moléculas de etanol detectadas na estrutura cristalina do complexo bem como da ocorrência de ligações de hidrogênio. As bandas referentes aos estiramentos vibracionais $(\mathrm{C}=\mathrm{N}) 1600 \mathrm{~cm}^{-1} \mathrm{e}(\mathrm{C}=\mathrm{S}) 748 \mathrm{~cm}^{-1}$ apresentam pequenas modificações em seus números de onda quando comparadas ao ligante livre, evidenciando o envolvimento destes átomos com a coordenação ao centro metálico. Os principais números de onda atribuídos aos compostos são apresentados na Tabela 1 .

\section{Estruturas cristalinas}

As Figuras 1 e 2 mostram as estruturas do ligante isatina-3-( $\mathrm{N}^{4}$ benziltiossemicarbazona) e do complexo com $\mathrm{Hg}(\mathrm{II})$, respectivamente.
Tabela 1. Principais bandas atribuídas a vibrações no ligante isatina-3-( $\mathrm{N}^{4}-$ benziltiossemicarbazona) e no complexo de $\mathrm{Hg}$ (II)

\begin{tabular}{lcccc}
\hline Compostos & $v(\mathrm{~N}-\mathrm{H}) \mathrm{cm}^{-1}$ & $v(\mathrm{C}=\mathrm{O}) \mathrm{cm}^{-1}$ & $v(\mathrm{C}=\mathrm{N}) \mathrm{cm}^{-1}$ & $v(\mathrm{C}=\mathrm{S}) \mathrm{cm}^{-1}$ \\
\hline $\mathrm{L}$ & $3298-3178(\mathrm{~m})$ & $1693(\mathrm{~F})$ & $1593(\mathrm{~F})$ & $744(\mathrm{~m})$ \\
$\mathrm{HgL}_{2}$ & $3425-3228(\mathrm{~m})$ & $1703(\mathrm{MF})$ & $1600(\mathrm{~F})$ & $748(\mathrm{~m})$ \\
\hline
\end{tabular}

Intensidades das bandas: $\mathrm{m}=$ média; $\mathrm{F}=$ forte; $\mathrm{MF}=$ muito forte

Tabela 2. Dados cristalográficos e refinamento estrutural isatina-3-( $\mathrm{N}^{4}$ benziltiossemicarbazona) e seu complexo de $\mathrm{Hg}$ (II)

\begin{tabular}{lcc}
\hline & Ligante & Complexo \\
\hline Fórmula Empírica & $\mathrm{C}_{15} \mathrm{H}_{12} \mathrm{~N}_{4} \mathrm{O} \mathrm{S}$ & $\mathrm{C}_{32} \mathrm{H}_{28} \mathrm{Hg} \mathrm{N}_{8} \mathrm{O}_{3} \mathrm{~S}_{2}$ \\
Peso Molecular & $296,35 \mathrm{~g} / \mathrm{mol}$ & $837,33 \mathrm{~g} / \mathrm{mol}$ \\
Temperatura & $295(2) \mathrm{K}$ & $295(2) \mathrm{K}$ \\
Comprimento de onda & $0,71073 \AA$ & $0,71073 \AA$
\end{tabular}

dos raios-X $\left(\mathrm{K}_{\alpha} \mathrm{Mo}\right)$

Sistema cristalino e grupo Monoclinico $/ P 2_{I} / c \quad$ Triclinico $/ P(-1)$ espacial

\begin{tabular}{|c|c|c|}
\hline $\begin{array}{l}\text { Dimensões da célula } \\
\text { unitária }\end{array}$ & $\begin{array}{c}a=6,3227(2) \AA \\
b=15,7973(7) \AA \\
c=14,4572(6) \AA \\
\alpha=90^{\circ} \\
\beta=93,9330(10)^{\circ} \\
\gamma=90^{\circ}\end{array}$ & $\begin{array}{c}a=9,5983(2) \AA \\
b=13,5747(3) \AA \\
c=14,3403(3) \AA \\
\alpha=66,775(10)^{\circ} \\
\beta=71,9930(10)^{\circ} \\
\gamma=78,8850(10)^{\circ}\end{array}$ \\
\hline $\begin{array}{l}\mathrm{Z} \text { (número de moléculas } \\
\text { por cela) }\end{array}$ & 4 & 2 \\
\hline Volume & $1440,61(10) \AA^{3}$ & $1627,56(6) \AA^{3}$ \\
\hline Densidade calculada & $1,366 \mathrm{mg} / \mathrm{m}^{3}$ & $1,709 \mathrm{mg} / \mathrm{m}^{3}$ \\
\hline Coeficiente de absorção & $0,228 \mathrm{~mm}^{-1}$ & $4,904 \mathrm{~mm}^{-1}$ \\
\hline $\mathrm{F}(000)$ & 616 & 824 \\
\hline Dimensão do cristal & $0,38 \times 0,12 \times 0,10 \mathrm{~mm}^{3}$ & $0,22 \times 0,17 \times 0,07 \mathrm{~mm}^{3}$ \\
\hline $\begin{array}{l}\text { Intervalo de } \theta \text { para coleta } \\
\text { de dados }\end{array}$ & 2,58 a $29,95^{\circ}$ & 2,67 a $30,07^{\circ}$ \\
\hline $\begin{array}{l}\text { Limites dos índices de } \\
\text { Miller }\end{array}$ & $\begin{array}{c}-8 \leq h \leq 7,-22 \leq k \leq 22 \\
-20 \leq l \leq 20\end{array}$ & $\begin{array}{c}-13 \leq h \leq 10,-19 \leq k \leq 18 \\
-20 \leq l \leq 20\end{array}$ \\
\hline $\begin{array}{l}\text { Reflexões coletadas/ } \\
\text { únicas }\end{array}$ & $\begin{array}{c}15765 / 4174 \\
{[\mathrm{R}(\text { int })=0,0305]}\end{array}$ & $\begin{array}{c}30333 / 9482 \\
{[\mathrm{R}(\text { int })=0,0274]}\end{array}$ \\
\hline $\begin{array}{l}\text { Completância para } \theta= \\
24,95\end{array}$ & $99,9 \%$ & $99,1 \%$ \\
\hline Min. e Máx. transmissão & 0,9775 e 0,9182 & 0,7253 e 0,4118 \\
\hline Método de refinamento & $\begin{array}{l}\text { Matriz de mínimos qua- } \\
\text { drados completa em } \mathrm{F}^{2}\end{array}$ & $\begin{array}{l}\text { Matriz de mínimos qua- } \\
\text { drados completa em } \mathrm{F}^{2}\end{array}$ \\
\hline $\begin{array}{l}\text { Dados/restrições/ } \\
\text { parâmetros }\end{array}$ & 4174 / 0 / 203 & 9482 / 0 / 432 \\
\hline Concordância sobre $\mathrm{F}^{2}$ & 0,849 & 1,012 \\
\hline $\begin{array}{l}\text { Índices R para os dados } \\
{[\mathrm{I}>2 \sigma(\mathrm{I})]}\end{array}$ & $\begin{array}{l}R 1=0,0482, \\
w R 2=0,1658\end{array}$ & $\begin{array}{l}R 1=0,0279, \\
w R 2=0,0534\end{array}$ \\
\hline $\begin{array}{l}\text { Índices R para todos os } \\
\text { dados }\end{array}$ & $\begin{array}{l}R 1=0,0953 \\
w R 2=0,2227\end{array}$ & $\begin{array}{l}R 1=0,0497 \\
w R 2=0,0590\end{array}$ \\
\hline Alturas de picos residuais & $0,310 \mathrm{e}-0,525 \mathrm{e} . \AA^{-3}$ & $0,544 \mathrm{e}-0,645 \mathrm{e} . \AA^{-3}$ \\
\hline Código no CCDC & 753879 & 753878 \\
\hline
\end{tabular}

As distâncias das ligações N-N [1,341(2) Å], C=S [1,651(2) ̊̊] e C-N[1,383(3) $\AA$ ] do ligante livre são alteradas quando o ligante se apresenta na forma aniônica coordenado ao centro metálico. As ligações N-N [1,355(2) e 1,354(2) Å] e C-S [1,741(2); 1,742(2) $\AA$ ] do complexo sofrem um pequeno alongamento e as ligações $\mathrm{C}-\mathrm{N}[1,326(3), 1,318(3) \AA]$ um encurtamento quando comparadas às ligações do ligante livre. Estas alterações evidenciam a deslocalização de carga ao longo da cadeia tiossemicarbazona (N-N-C-S) do ligante desprotonado, ou seja, apresenta valores intermediários entre os es- 
Tabela 3. Principais distâncias $(\AA)$ e ângulos de ligação $\left(^{\circ}\right)$ para as estruturas de isatina-3-( $\mathrm{N}^{4}$-benziltiossemicarbazona) e seu complexo de $\mathrm{Hg}(\mathrm{II})$

\begin{tabular}{|c|c|c|c|}
\hline \multicolumn{4}{|l|}{ Ligante } \\
\hline $\mathrm{C}(11)-\mathrm{N}(11)$ & $1,293(2)$ & $\mathrm{N}(11)-\mathrm{C}(11)-\mathrm{C}(12)$ & $127,23(18)$ \\
\hline $\mathrm{C}(11)-\mathrm{C}(12)$ & $1,505(2)$ & $\mathrm{O}(1)-\mathrm{C}(12)-\mathrm{N}(18)$ & $127,53(17)$ \\
\hline $\mathrm{C}(12)-\mathrm{O}(1)$ & $1,231(2)$ & $\mathrm{N}(11)-\mathrm{N}(12)-\mathrm{C}(1)$ & $120,27(15)$ \\
\hline $\mathrm{C}(12)-\mathrm{N}(18)$ & $1,358(3)$ & $\mathrm{C}(1)-\mathrm{N}(1)-\mathrm{C}(41)$ & $133,05(18)$ \\
\hline $\mathrm{N}(11)-\mathrm{N}(12)$ & $1,341(2)$ & $\mathrm{N}(1)-\mathrm{C}(1)-\mathrm{S}(1)$ & $129,73(15)$ \\
\hline $\mathrm{C}(1)-\mathrm{N}(12)$ & $1,383(3)$ & $\mathrm{C}(12)-\mathrm{N}(18)-\mathrm{C}(17)$ & $111,83(16)$ \\
\hline $\mathrm{C}(1)-\mathrm{N}(1)$ & $1,333(2)$ & $\mathrm{O}(1)-\mathrm{C}(12)-\mathrm{C}(11)$ & $126,94(17)$ \\
\hline$C(1)-S(1)$ & $1,651(2)$ & $\mathrm{N}(1)-\mathrm{C}(1)-\mathrm{N}(12)$ & $112,87(17)$ \\
\hline $\mathrm{C}(41)-\mathrm{N}(1)$ & $1,417(2)$ & & \\
\hline$C(11)-C(19)$ & $1,450(3)$ & & \\
\hline \multicolumn{4}{|l|}{ Complexo } \\
\hline $\mathrm{N}(13)-\mathrm{Hg}$ & $2,389(2)$ & $\mathrm{N}(13)-\mathrm{Hg}-\mathrm{S}(21)$ & $129,17(5)$ \\
\hline $\mathrm{N}(23)-\mathrm{Hg}$ & $2,4298(19)$ & $\mathrm{N}(13)-\mathrm{Hg}-\mathrm{N}(23)$ & $130,32(6)$ \\
\hline $\mathrm{S}(11)-\mathrm{Hg}$ & $2,4465(7)$ & $\mathrm{S}(21)-\mathrm{Hg}-\mathrm{N}(23)$ & $76,27(5)$ \\
\hline $\mathrm{S}(21)-\mathrm{Hg}$ & $2,4211(7)$ & N(13)-Hg-S(11) & $76,79(5)$ \\
\hline $\mathrm{C}(1)-\mathrm{N}(12)$ & $1,326(3)$ & $\mathrm{S}(21)-\mathrm{Hg}-\mathrm{S}(11)$ & $133,79(3)$ \\
\hline $\mathrm{C}(1)-\mathrm{N}(11)$ & $1,358(3)$ & $\mathrm{N}(23)-\mathrm{Hg}-\mathrm{S}(11)$ & $118,72(5)$ \\
\hline $\mathrm{C}(1)-\mathrm{S}(11)$ & $1,722(3)$ & $\mathrm{N}(12)-\mathrm{C}(1)-\mathrm{S}(11)$ & $130,03(18)$ \\
\hline $\mathrm{C}(2)-\mathrm{N}(22)$ & $1,318(3)$ & $\mathrm{N}(11)-\mathrm{C}(1)-\mathrm{S}(11)$ & $114,27(19)$ \\
\hline $\mathrm{C}(2)-\mathrm{N}(21)$ & $1,362(3)$ & $N(22)-C(2)-S(21)$ & $129,78(18)$ \\
\hline$C(2)-S(21)$ & $1,741(2)$ & $\mathrm{N}(21)-\mathrm{C}(2)-\mathrm{S}(21)$ & $111,68(18)$ \\
\hline $\mathrm{C}(31)-\mathrm{C}(32)$ & $1,505(3)$ & $\mathrm{O}(11)-\mathrm{C}(32)-\mathrm{C}(31)$ & $127,3(2)$ \\
\hline $\mathrm{C}(32)-\mathrm{O}(11)$ & $1,223(3)$ & $\mathrm{O}(11)-\mathrm{C}(32)-\mathrm{N}(33)$ & $127,1(2)$ \\
\hline $\mathrm{C}(32)-\mathrm{N}(33)$ & $1,356(4)$ & $\mathrm{O}(21)-\mathrm{C}(62)-\mathrm{C}(61)$ & $126,9(2)$ \\
\hline $\mathrm{C}(31)-\mathrm{N}(13)$ & $1,292(3)$ & $\mathrm{O}(21)-\mathrm{C}(62)-\mathrm{N}(63)$ & $126,8(2)$ \\
\hline $\mathrm{C}(62)-\mathrm{N}(63)$ & $1,353(3)$ & $\mathrm{C}(1)-\mathrm{N}(12)-\mathrm{N}(13)$ & $114,77(19)$ \\
\hline $\mathrm{C}(61)-\mathrm{N}(23)$ & $1,292(3)$ & $\mathrm{C}(31)-\mathrm{N}(13)-\mathrm{N}(12)$ & $115,84(19)$ \\
\hline$C(61)-C(62)$ & $1,500(3)$ & $\mathrm{C}(2)-\mathrm{N}(22)-\mathrm{N}(23)$ & $114,39(19)$ \\
\hline $\mathrm{C}(62)-\mathrm{O}(21)$ & $1,227(3)$ & $\mathrm{C}(61)-\mathrm{N}(23)-\mathrm{N}(22)$ & $117,70(19)$ \\
\hline $\mathrm{N}(22)-\mathrm{N}(23)$ & $1,355(2)$ & & \\
\hline $\mathrm{N}(12)-\mathrm{N}(13)$ & $1,354(2)$ & & \\
\hline
\end{tabular}

perados para ligações simples e duplas, justificada pelo envolvimento dos átomos com a coordenação ao centro metálico.

$\mathrm{Na}$ unidade assimétrica do complexo, o ligante isatina-3-( $\mathrm{N}^{4}$ benziltiossemicarbazona) apresenta-se desprotonado na posição $\mathrm{N}^{2}$ e o íon $\mathrm{Hg}(\mathrm{II})$ está coordenado via $\mathrm{N}^{1}$ (azometínico) e $\mathrm{S}$ doador formando anéis quelatos de cinco membros. As ligações $\mathrm{Hg}-\mathrm{N} 13$

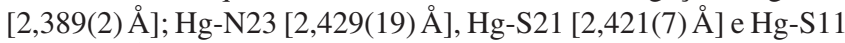
$[2,446(7) \AA]$ que completam a esfera de coordenação do $\mathrm{Hg}$ (II), são bastante similares às distâncias observadas para outros complexos de mercúrio com ligantes semelhantes. ${ }^{18}$

A molécula do ligante isatina-3-( $\mathrm{N}^{4}$-benziltiossemicarbazona) é praticamente plana, os fragmentos [C17-C19N18C12C11N11]/ [N12C1N1C41-C46] apresentam um ângulo de desvio da planaridade de $2,47(0,06)^{\circ}$; com um desvio médio quadrático de $(\mathrm{rms}=0,0166)$ e $(\mathrm{rms}=0,0152)$ respectivamente. Ao coordenaremse ao centro metálico as moléculas do ligante mantêm a planaridade e atuam de forma bidentada, levando a uma geometria de coordenação tetraédrica distorcida. A literatura descreve uma estrutura com o íon $\mathrm{Hg}$ (II) tetraédrico, porém com um ligante tiossemicarbazona quelato e dois iodetos monodentados. ${ }^{19}$ Os ângulos que descrevem a esfera de coordenação são: $\mathrm{N}(23)-\mathrm{Hg}$ $\mathrm{S}(11) 118,72(5)^{\circ}, \mathrm{N}(13)-\mathrm{Hg}-\mathrm{S}(21) 129,17(5)^{\circ}, \mathrm{S}(21)-\mathrm{Hg}-\mathrm{N}(23)$ $76,27(5)^{\circ}, \mathrm{N}(13)-\mathrm{Hg}-\mathrm{S}(11) 76,79(5)^{\circ}$, que diferem significativamente dos ângulos do tetraedro em função das tensões angulares

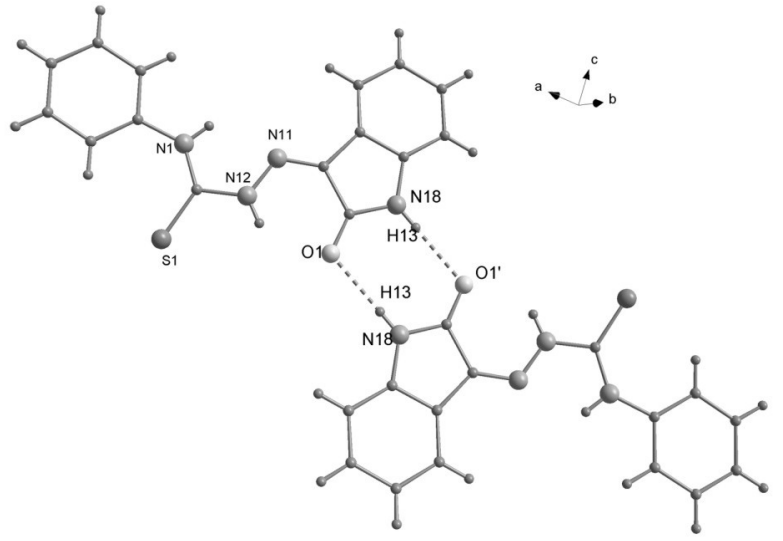

Figura 3. Estrutura dimérica de isatina-3-( $N^{4}$-benziltiossemicarbazona) formada por ligações de hidrogênio. Código de simetria ('): 4-x, 1-y, 1-z

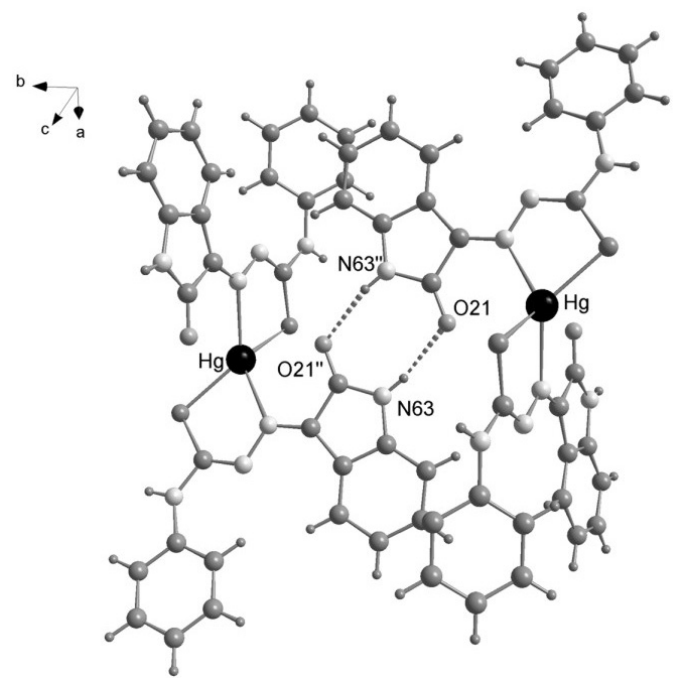

Figura 4. Estrutura dimérica de bis[isatina-3-( $N^{4}$-benziltiossemicarbazonato)] mercúrio (II) formada por ligações de hidrogênio. Código de simetria: $i=-x, 1-y, 1-z$

impostas pelo modo de coordenação quelato e volume dos anéis terminais das moléculas dos ligantes.

Os dados cristalográficos e os principais ângulos e distâncias de ligação das estruturas estão reunidos nas Tabelas 2 e 3, respectivamente.

Em ambos os compostos, observam-se importantes interações intermoleculares com formação de estruturas diméricas. As ligações de hidrogênio presentes na estrutura do ligante (Figura 3) interrelacionam

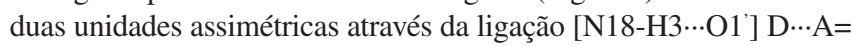
2,907(2) Å, ângulo 177(2) ${ }^{\circ}$, código de simetria ('): 4-x, 1-y, 1-z.

As ligações de hidrogênio no complexo de mercúrio levam a uma estrutura dimérica (Figura 4) com ligações do tipo [N(63)$\left.\mathrm{H}(4) \cdots \mathrm{O}(21)^{\mathrm{i}}\right] \mathrm{D}-\mathrm{A}=2,863(3) \AA$, ângulo $164(3)^{\circ}$. Código de simetria: $\mathrm{i}=-\mathrm{x}, 1-\mathrm{y}, 1-\mathrm{z}$.

\section{CONCLUSÕES}

Pode-se concluir que ligantes derivados de isatina e tiossemicarbazidas são excelentes agentes quelantes. Atuam na forma aniônica com deslocalização de carga eletrônica ao longo da cadeia tiossemicarbazona mantendo-se praticamente planos. Frente ao íon $\mathrm{Hg}$ (II) o modo preferencial de coordenação é o bidentado N, S doador, levando a uma geometria tetraédrica distorcida e não ao modo tridentado amplamente encontrado na literatura. Tanto a estrutura 
do ligante quanto a estrutura do complexo apresentam interações intermoleculares levando à formação de dímeros.

\section{AGRADECIMENTOS}

Ao CNPq, à FAPERGS e CAPES pelo suporte financeiro e bolsas.

\section{REFERENCIAS}

1. Puntel, G. O.; Carvalho, N. R.; Gubert, P.; Palma, A. S.; Corte, C. L.; Ávila, D. S.; Pereira, M. E.; Carratu, V. S.; Bresolin, L.; Rocha, J. B.; Soares, F. A. A.; Chem. Biol. Interact. 2009, 177, 153.

2. Beraldo, H. de O.; Quim. Nova 2004, 27, 461.

3. Bermejo, E.; Castiñeiras, A.; Domínguez, R.; Carballo, R.; MaichleMössmer, C.; Strähle ,J.; West, D. X.; Z. Anorg. Allg. Chem. 1999, 625, 961.

4. Sagdinc, S.; Köksoy, B.; Kandemirli, F.; Bayari, S. H.; J. Mol. Struct. 2009, 917, 63.

5. Bal, T. R.; Anand B.; Yogeeswari, P.; Sriram, D.; Bioorg. Med. Chem. Lett. 2005, 15, 4451.

6. Sebastian, L.; Desai, A.; Shampur, M. N.; Perumal, Y.; Sriram, D.; Vasanthapuram, R.; Virology J. 2008, 5, 64.

7. Konstantinovic, S. S.; Radovanovic, B. C.; Krkljes, A.; J. Therm. Anal. Calorim. 2007, 90, 525 .

8. Du, K.; Liu, S.; J. Mol. Struct. 2008, 874, 138.
9. Lobana, T. S.; Khanna, S.; Sharma, R.; Hundal, G.; Sultana, R.; Chaudhary, M.; Butcher, R. J.; Castñeiras, A.; Cryst. Growth Des. 2008, $8,1203$.

10. Vatsa, G.; Pandey, O. P.; Sengupta, S. K.; Bioinorg. Chem. Appl. 2005, 3,151 .

11. Sheldrick, G. M.; SHELXL-97; Program for Crystal Structure Refinement, Universidade de Göttingen, Alemanha, 1997.

12. Sheldrick, G. M.; SADABS, Program for Empirical Absorption Correction of Area Detector Data, Universidade de Göttingen, Alemanha, 1996.

13. Ivanov, V. E.; Tihomirova, N. G.; Tomchin, A. B.; Zh. Obshch. Khim. 1988, 58, 2737.

14. Akinchan, N. T.; Drozdzewski, P. M.; Holzer, W.; J. Mol. Struct. 2002, $641,17$.

15. Silverstein, R. M.; Webster, F. X.; Kiemle, D. J.; Identificação Espectrométrica de Compostos Orgânicos, $7^{\mathrm{a}}$ ed., LTC: Rio de Janeiro, 2007.

16. Samus, N. M.; Tsapkov, V. I.; Gulya, A. P.; Russ. J. Gen. Chem. 2004, $74,1428$.

17. Colthup, N. B.; Daly, L. H.; Wieberly, S. E.; Introduction to Infrared and Raman Spectroscopy, Academic Press: New York, 1990.

18. Bermejo, E.; Castiñeiras, A.; Santos, I. G.; West, D. X.; Z. Anorg. Allg. Chem. 2004, 630, 1096.

19. Fan, Y. J.; Ma, J. P.; Sun, Z. X.; Acta Crystallogr., Sect. E: Struct. Rep. Online 2007, 63, m2044. 